\title{
Impact of the NSSP's transition to ESSENCE on chief complaint field-based syndromes
}

\author{
Rasneet S. Kumar* and Jessica R. White \\ Maricopa County Department of Public Health, Office of Epidemiology, Phoenix, AZ, USA
}

\section{Objective}

To evaluate the effect and implications of changing the chief complaint field during the National Syndromic Surveillance Program (NSSP) transition from BioSense 2.0 analytical tools to BioSense Platform - ESSENCE

\section{Introduction}

In January 2017, the NSSP transitioned their BioSense analytical tools to Electronic Surveillance System for Early Notification of Community-Based Epidemics (ESSENCE). The chief complaint field in BioSense 2.0 was a concatenation of the record's chief complaint, admission reason, triage notes, and diagnostic impression. Following the transition to ESSENCE, the chief complaint field was comprised of the first chief complaint entered or the first admission reason, if the chief complaint was blank. Furthermore, the ESSENCE chief complaint field was electronically parsed (i.e., the original chief complaint text was altered to translate abbreviations and remove punctuation). This abstract highlights key findings from Maricopa County Department of Public Health's evaluation of the new chief complaint field, its impact on heat-related illness syndromic surveillance, and implications for ongoing surveillance efforts.

\section{Methods}

For this evaluation, we used the heat-related illness query recommended in Council of State and Territorial Epidemiologists' (CSTE) 2016 Guidance Document for Implementing Heat-Related Illness Syndromic Surveillance. Before the transition, we used BioSense 2.0's, phpMyAdmin analytical tool to generate a list of patients who visited Maricopa County emergency departments or inpatient hospitals between 5/1/2016 - 9/30/2016 due to heat-related illness. After the transition, we used the CC and DD Category "Heatrelated Illness, $v 1$ " in ESSENCE, which was based on the CSTE heat-related illness query, to generate a list of patients for the same time period. We compared the line-lists and time-series trends from phpMyAdmin and ESSENCE.

\section{Results}

The phpMyAdmin analytical tool identified 785 heat-related illness records with the query (Figure). $642(82 \%)$ of these heatrelated illness records were also captured by ESSENCE. Reasons for $143(18 \%)$ records not being identified by ESSENCE included: the patient's admission reason field contained keywords that were not available in the ESSENCE chief complaint field ( $\mathrm{n}=94,66 \%)$; data access changed, which disabled access to patients who resided in zip codes that crossed a county border $(30,21 \%)$; discrepancies between ESSENCE parsing and text in the original chief complaint $(11,8 \%)$; heat-related illness discharge diagnoses were removed by the facility after the phpMyAdmin line-list for heat-related illness was extracted (7, 5\%); and one record was undetermined. Conversely, ESSENCE captured 36 additional heat-related illness records, not previously captured by phpMyAdmin. Reasons included: a query exclusion term was located in the patient's admission reason but not the ESSENCE chief complaint field $(16,44 \%)$; a heat-related illness discharge diagnosis code was added by the facility after the data were extracted by phpMyAdmin $(4,11 \%)$; and 16 (44\%) were undetermined. Time-series trend evaluation revealed a significant correlation between the two surveillance tools (Pearson coefficient $=0.97, \mathrm{p}<0.01)$.

\section{Conclusions}

Though the data trends over time were not significantly affected by changes in the chief complaint field, differences in the field's composition have important implications for syndromic surveillance practitioners. Free-text queries designed to search the chief complaint field in ESSENCE may not retrieve records previously identified with BioSense 2.0 analytical tools, which may limit individual casefinding capacity. The elimination of admission reason from the chief complaint field in ESSENCE has the greatest effect on case-finding capacity. Furthermore, surveillance reports produced by ESSENCE cannot be directly compared to reports that were previously published with data from BioSense 2.0. These limitations may be addressed if ESSENCE creates a feature that allows users to easily query fields (e.g., admission reason) in addition to the chief compliant field.

Figure. Maricopa County, Arizona heat-related illness syndromic surveillance results identified by
BioSense 2.0 phpMyAdmin and BioSense Platform ESSENCE, 5/1/2016-9/30/2016

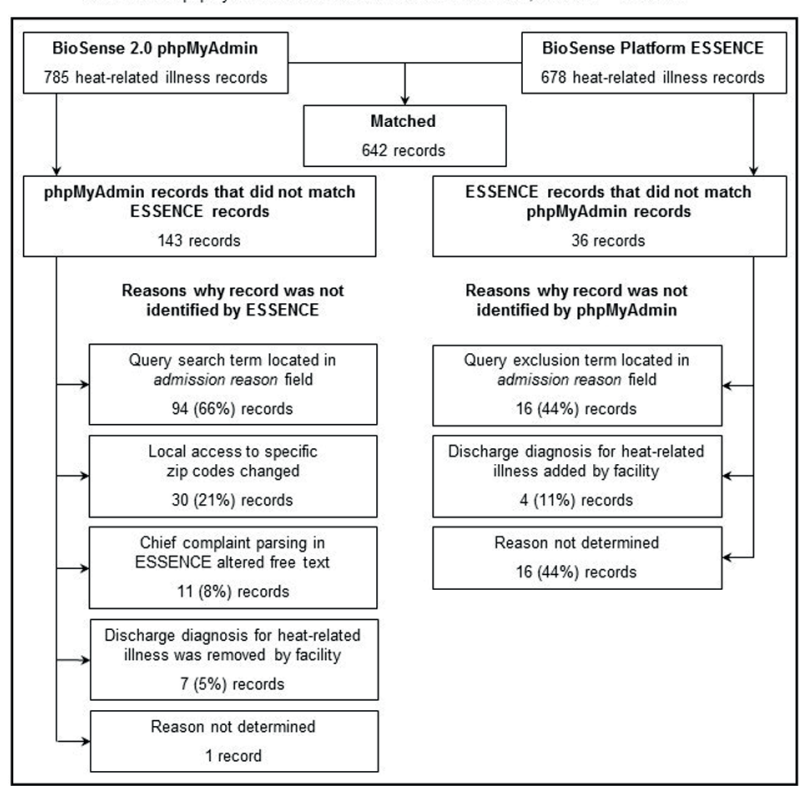

Keywords

ESSENCE; Chief Complaint; Transition; Evaluation; Heat-related Illness

\section{Acknowledgments}

The authors thank the Arizona Department of Health Services for contributions

\section{${ }^{*}$ Rasneet S. Kumar}

E-mail: rasneetkumar@mail.maricopa.gov 